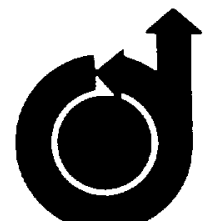

AIAA 80-1628R

Criteria for Side-Force Control in Air-to-Ground

Target Acquisition and Tracking

R.I. Sammonds, W.E. McNeill, J.W. Bunnell

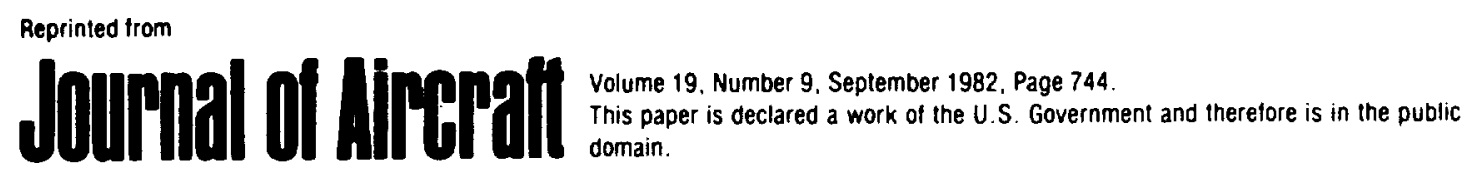




\title{
NASATM- $80-208078$ \\ Criteria for Side-Force Control in Air-to-Ground Target Acquisition and Tracking
}

\author{
Robert 1. Sammonds* and Walter E. McNeill* \\ NASA Ames Research Center, Moffell Field, Calif. \\ and \\ John W. Bunnell $†$ \\ Air Force Wright Aeronautical Laboratories, WPAFB, Ohio
}

\begin{abstract}
A moving-base simulator experiment conducted at Ames Research Center demonstrated that a wings-levelturn control mode improved flying qualities for air-to-ground weapons delivery compared with those of a conventional aircraft. Evaluations of criteria for dynamic response for this system have shown that pilot ratings correlate well with equivalent time constant of the initial response and with system bandwidth. Ranges of this time constant, as well as digital-system transport delays and lateral-acceleration control authorities that encompassed level I through level IIl handling qualities, were delermined.
\end{abstract}

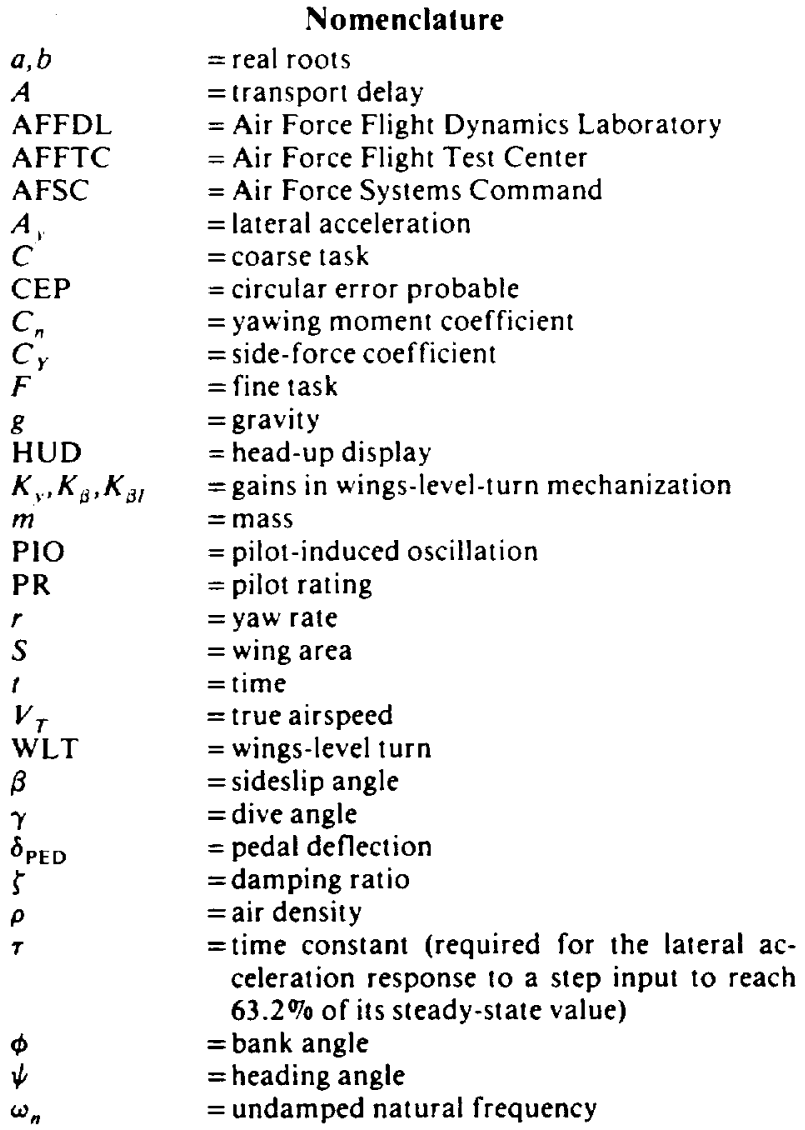

\section{Introduction}

D IVE bombing is the most common way of delivering free-fall, non-nuclear weapons against ground targets. With respect to the low-level attack mode, it offers the ad-

Presented as Paper 80-1628 at the AlAA 7th Atmospheric Flight Mechanics Conference, Danvers, Mass., Aug. 11-13, 1980; submitted Oct. 8, 1980; revision received Nov. 6, 1981. This paper is declared a work of the U.S. Government and therefore is in the public domain.

- Aerospace Engineer.

†Aerospace Engineer, Flight Dynamics Laboratory. vantages of better target acquisition, reduced vulnerability to certain types of hostile ground fire, and delivery of largeyield, low-drag weapons. However, the delivery variables (airspeed, altitude, and attitude) are not as easily at tainable as in low-level bombing and the attack is often less accurate. To score a direct hit, the aircraft must arrive at a particular point is space with the correct airspeed, dive angle, and $g$ loading and with proper corrections made for the existing wind conditions.

Motivations for improving the dive-bombing task are threefold: 1) to increase the aiming accuracy; 2) to decrease pilot workload; and 3) to decrease aircraft vulnerability by decreasing the time to acquire the target, aim, and launch the weapon.

Previous investigations ${ }^{1.9}$ have shown that certain advanced control modes, which increase aircraft agility and the preciseness of maneuvers, can be used to provide a large increase in the combat potential of conventional aircraft. One of the most promising of these advanced control modes for use in the dive-bombing task ${ }^{3}$ is the wings-level turn (WLT). This mode permits a heading change by commanding a lateral acceleration while holding the wings level $(\phi=0)$ and maintaining a zero sideslip $(\beta=0)$. This maneuver eliminates the pendulum motion of the fixed depressed reticle sight (pipper) that occurs during rolling maneuvers when the aircraft's roll axes and the sight do not coincide. Elimination of the pendulum motion allows for a more rapid and accurate acquisition of the target than can be accomplished with a conventional airplane, thus reducing the time over the target by permitting increased delivery airspeeds.

Although fight and simulation data exist to show the potential advantages of WLT capability, there is a lack of systematic research on the flying-qualities criteria required for use in design of this control mode. The purposes of this present research are 1) to conduct a systematic parametric investigation of the variables affecting the performance of an aircraft during an air-to-ground weapon delivery task in which the WLT control mode is used and 2) to compare the results with those for a conventional, current-generation (bank-to-turn) fighter aircraft. This program was conducted in the six-degrees-of-freedom Flight Simulator for Advanced Aircraft (FSAA) at Ames Research Center. Evaluations were obtained for a range of equivalent system dynamic characteristics, digital transport delays, and control authorities. Results are presented in this paper in the form of pilot ratings and commentary and control usage. 


\section{Simulation Test Program}

\section{Description of Simulator}

This investigation was conducted using the six-degrees-offreedom Flight Simulator for Advanced Aircraft (FSAA) shown in Fig. 1. This simulator, described in Ref. 4, was equipped to represent a fighter cockpit with a center stick, all necessary instrumentation, and a head-up display. Hydraulically actuated control loaders on all three axes were programmed to give the cockpit control force-feel characteristics typical of an advanced fighter aircraft.

The pilot in the cab was provided visual and aural cues, as well as motion cues. The visual cues consisted of a bull's eye target located on a terrain board and displayed on a color TV monitor; the scene was viewed through a collimating lens mounted above the instrument panel. The visual scene was generated by a computer-driven, six-degrees-of-freedom TV camera that duplicated the aircraft motion with respect to the dive-bombing task, but restricted the pilot to a forward view. Scale buildings were located near the target to add realism. The visual display system (see VFA-07. Table 4.2.1-1 in Ref. 4) was modified in the pitch plane by biasing the pitch prism to obtain the necessary look-down capability for the divebombing task. The maximum pitch thus provided was +10 , $-40 \mathrm{deg}$, which effectively limited the desired dive angle for the bombing runs to $-30 \mathrm{deg}$. Aural cues consisted of engine noise modulated by engine rpm and introduced into the cab through stereo speakers.

\section{Modeling}

A conventional six-degrees-of-freedom mathematical model was developed to represent a state-of-the-art fighter

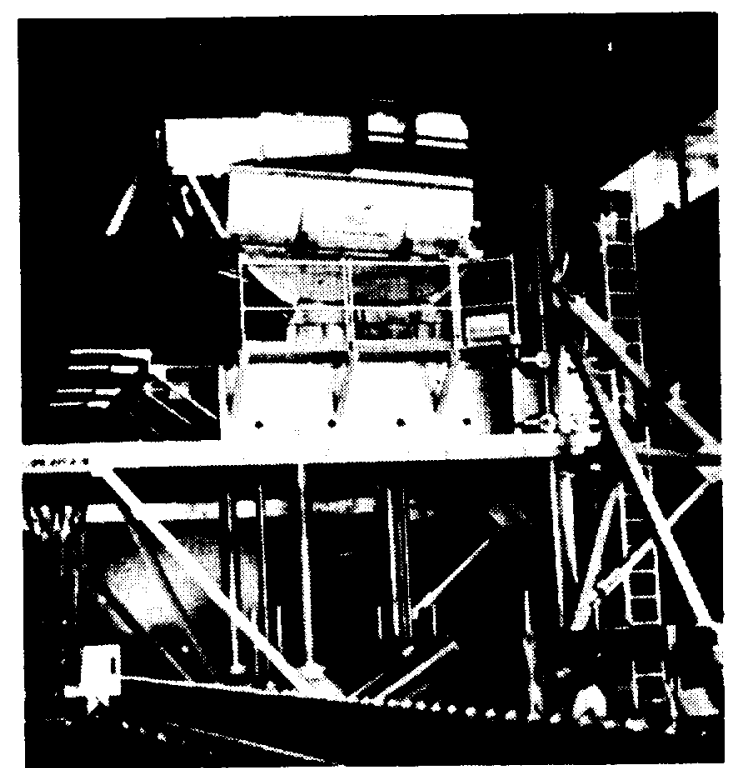

Fig. 1 A mes Flight Simulator for Advanced Aircraft (FSAA). aircraft. This model was used as the baseline aircraft and had flying qualities similar to those of the F-15.

The WLT flight-control mode was modeled as a transfer function, relating the lateral acceleration $A_{y}$ to a rudder pedal deflection, of the form

$$
\frac{A_{y}}{\delta_{\mathrm{PED}}}=\frac{\left(K_{y} / 3.25\right) e^{-A s}}{s^{2} / \omega_{n}^{2}+2 \zeta s / \omega_{n}+1}
$$

The yaw rate required for a zero-sideslip turn was calculated from the commanded $A_{v}$. A proportional-plusintegral sideslip angle feedback was included to ensure minimal sideslip. The commanded lateral acceleration was implemented in the simulation by using the calculated $C_{Y}$ in the aerodynamic equations. Similarly, the calculated yaw rate (including feedback terms) was used directly in the equations of motion. The block diagram in Fig. 2 shows how the WLT mode was mechanized for the simulation. Although this technique did not simulate any real aircraft, it did facilitate the variation of important flying-qualities parameters and allowed the study of pure uncoupled responses, thus justifying the idealized simulation.

\section{Test Conditions}

The gain, $K_{1}$, transport delay, $A$, natural frequency, $\omega_{n}$, and the damping ratio, $\zeta$, of the $\boldsymbol{A}_{1} / \delta_{\mathrm{PED}}$ transfer function were varied, either singly or in combination, during the experiment. The primary investigation was of the effect of the undamped natural frequency and damping ratio on the handling qualities of the WLT control mode. The matrix for these runs is shown in Table 1 for various values of bandwidth, with bandwidth defined as the frequency at which the amplitude of the Bode plot decreases by $3 \mathrm{~dB}$ from a steadystate condition (see sketch in Table 1).

Additional tests were made to evaluate the effect of adding various amounts of transport delay, $A$, 10 a system having good handling qualities, and three levels of commanded authority, $K_{y}$. The matrices for these programs are presented in Tables 2 and 3.

For all runs, the desired release conditions were a dive angle, $\gamma$, of $-30 \mathrm{deg}$, a velocity of $365.76 \mathrm{~m} / \mathrm{s}(1200 \mathrm{ft} / \mathrm{s})$, and an altitude of $1524 \mathrm{~m}(5000 \mathrm{ft})$. The high release velocity was determined from preliminary runs in conjunction with the initial and release altitudes because it resulied in a difficult task - one that could be accomplished with a good system but not with a poor one. The average time for each run from target acquisition to bomb drop was between 4 and $5 \mathrm{~s}$; the shorter time was for the alternative target maneuver.

\section{Task}

The test program was limited to an air-to-ground weapon delivery task using a fixed-depressed-reticle sight and an unguided bomb. The piloting task was to roll onto the target from a $90-\mathrm{deg}$ heading of fset at an altitude of $3048 \mathrm{~m}(10,000$ $\mathrm{ft}$ ), establish a - 30-deg dive angle, and release the bomb at a specified set of release conditions (airspeed and altitude). A schematic of this maneuver is shown in Fig. 3. Because the

Fig. 2 Wings-level-turn mechanization.
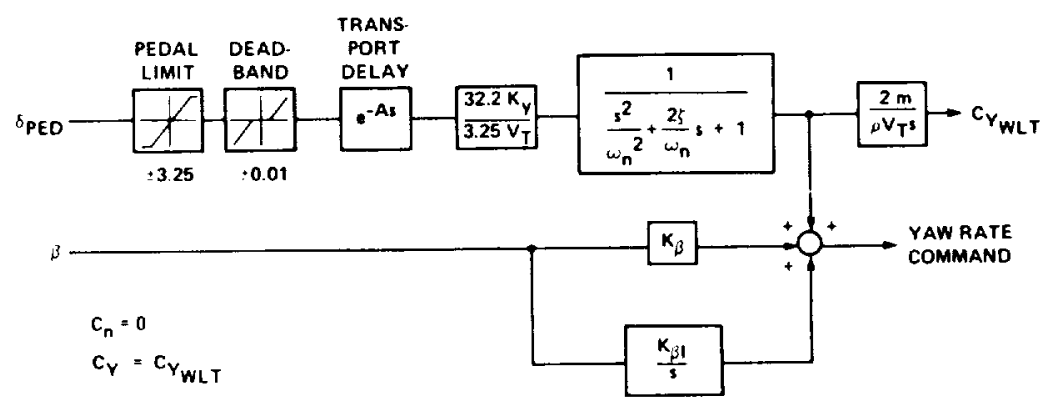
Table 1 Test matrix-transfer function bandwidth for combinations of frequency and damping ratio

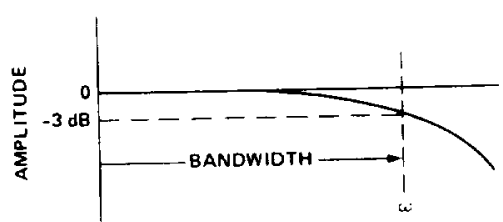

$\zeta$

\begin{tabular}{|c|c|c|c|c|c|c|}
\hline$\omega_{n}$ & 0.3 & 0.5 & 0.7 & 1.0 & 1.4 & 2.0 \\
\hline 0.5 & $0.71^{\mathrm{a}}$ & 0.64 & & & & \\
\hline 1 & 1.42 & 1.27 & 1.01 & 0.64 & 0.41 & 0.27 \\
\hline 2 & 2.84 & 2.54 & 2.02 & 1.29 & 0.82 & 0.53 \\
\hline 3 & 4.26 & 3.81 & 3.03 & 1.93 & 1.22 & 0.80 \\
\hline 4.5 & 6.39 & 5.72 & 4.55 & 2.90 & 1.84 & 1.20 \\
\hline 6 & & & 6.06 & 3.86 & 2,45 & 1.60 \\
\hline 8 & & & 8.08 & 5.15 & 3.26 & 2.13 \\
\hline 10 & & & & 6.44 & 4.08 & 2.67 \\
\hline 12 & & & & 7.72 & 4.90 & 3.20 \\
\hline 15 & & & & & 6.12 & 4.00 \\
\hline 19 & & & & & 7.75 & 5.07 \\
\hline 23 & & & & & & 6.13 \\
\hline 28 & & & & & & 7.46 \\
\hline
\end{tabular}

a Bandwidth frequency.

Table 2 Test matrix-transport delay

\begin{tabular}{llll}
\hline & $\omega_{n}$ & $\zeta$ & $A$ \\
\hline$A_{y}$ \\
$\delta_{\text {PED }}=\frac{\left(K_{y} / 3.25\right) e^{-A 5}}{s^{2} / \omega_{n}^{2}+2 \zeta s / \omega_{n}+1}$ & 15 & 1.4 & 0 \\
& 15 & 1.4 & 0.105 \\
& 15 & 1.4 & 0.24 \\
& 15 & 1.4 & 0.49 \\
\hline
\end{tabular}

Table 3 Tesi matrix $\rightarrow$ conirol authority

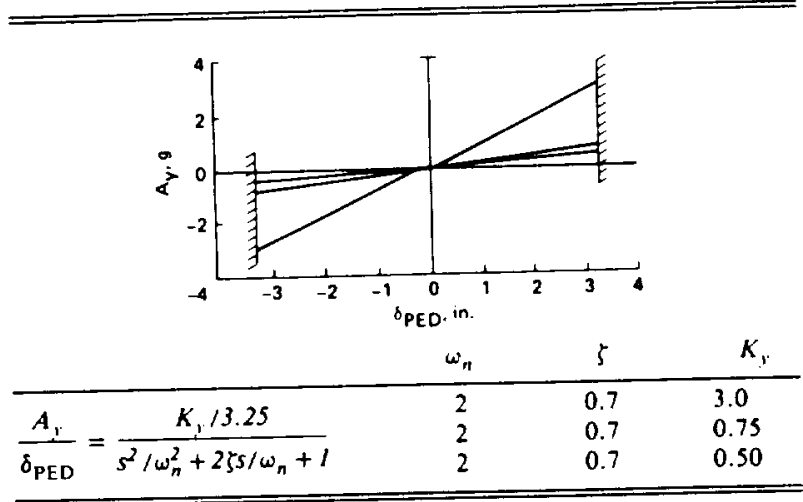

visual presentation in the cab did not provide for side-window viewing, the initial heading change and roll-in until target acquisition was an open-loop task that had to be learned by the pilots. A $4-6 \mathrm{~g}$ diving turn was required to align the pipper on the target and attain a -30 -deg dive angle. The pilots were given sufficient practice time to become adept at this maneuver.

The bull's-eye target located on the terrain board consisted of concentric circles that were $50,100,500,1000,1500$, and $2000 \mathrm{ft}$ (scale) in diameter. A normal run was made with respect to the center of the bull's-eye. However, in order to severely exercise the WLT capability of the aircraft, a secondary target, consisting of a large white dot, was located on the outer ring of the bull's-eye normal to the line of flight. Approximately $50 \%$ of the time, in a random manner, a light

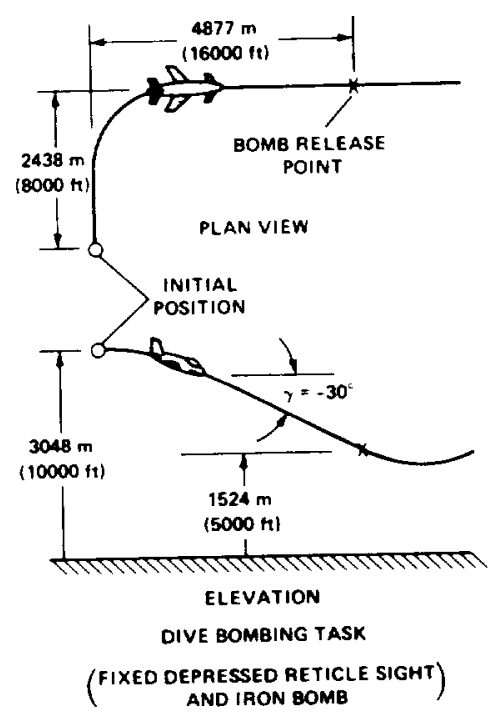

Fig. 3 Dive-bombing 1ask.

located at the center of the bull's-eye signaled the pilot to bomb the secondary target. This signal light was activated only after the pilot was aligned with the primary target, thus necessitating a $304.8-\mathrm{m}(1000-\mathrm{ft})$ lateral translation of the flight path. Bombing runs to the primary and secondary targets will be referred to in the future as the fine and coarse task, respectively. Although it is realized that this alternative maneuver is probably not representative of a real-life situation, it was selected as an easy way to subject the WLT mode to a severe translational maneuver in order to evaluate its gross maneuvering capabilities. A similar task could have been devised, using wind shears or gusts, but it was felt that this change-of-target maneuver would generate comparable results with less effort. The average time between the light signal and bomb drop was $4 \mathrm{~s}$.

\section{Data Acquisition}

The parametric evaluation of the WLT control mode was accomplished by (wo USAF pilots (A and B) from the 3246th 
Test Wing (AFSC), Eglin AFB, and by one pilot (C) from the USAF Test Pilots School, Edwards AFB. Each pilot made at least two runs at both the primary and secondary targets for each set of parameters being evaluated, with the targets being selected in a random manner. Each pilot was allowed to make as many runs as necessary for an accurate evaluation of the task. At the end of each set of runs, for a given parameter, the pilots were instructed to give pilot ratings for both the fine and coarse tasks, based on the Cooper-Harper rating scale of Ref. 5, giving reasons for their ratings, as well as comments on the flying qualities and their ability to accomplish the task. A maximum Cooper-Harper rating of 7 was established as a worst condition since there was never any danger of losing control of the aircraft.

The two pilots from Eglin AFB were responsible for the parametric evaluations listed in Table 1. Each of these pilots went through the matrix at least twice. Additional repetitions were made for points having a spread of more than one pilot rating until a consistent rating was obtained. The ratings were averaged to determine a single value for each parametric variation. The parameters in the matrix were selected randomly to avoid direct comparison with an adjacent point in the matrix. A baseline WLT condition having a natural frequency, $\omega_{n}$, of 4.5 and a damping ratio, $\zeta$, of 1.0 was specified and was used for all practice and training runs. Pilot comments could then be compared with this baseline configuration. Runs were also made with the baseline aircraft (no WLT) for comparison.

These same two pilots were also responsible for the evaluation of the matrix shown in Table 2; however, because of time limitations, they only went through this matrix once. The third pilot, the one from Edwards AFB, was responsible for the control authority evaluation of Table 3.

\section{Results and Discussion}

As a prelude to the parametric evaluation of the WLT control mode, several simulations were conducted to establish the baseline airplane configuration, the dive-bombing task, and the mechanization of the WLT control mode.

\section{Simulator Validation}

Validation of the baseline airplane configuration was based on the subjective assessment of a number of pilots from the Air Force Flight Test Center (AFFTC), Edwards AFB; Air Force Flight Dynamics Laboratory (AFFDL), WrightPatterson AFB; and Ames Research Center. These pilots were all experienced at flying modern fighter aircraft (F-4's, A-7's, F-15's, and T-38's) and with air-to-ground weapon delivery. Most were graduates of either the Air Force or Navy test pilots school. All agreed that the baseline configuration was a good representation of a state-of-the-art fighter aircraft with good flying qualities; the F-15 pilots felt it to be comparable to an F-15.

The dive-bombing task was thought to be satisfactory for the evaluation of the WLT control mode, although there was some misgiving because of the lack of side-window viewing. However, the open-loop task of acquiring the target from a 90-deg heading offset was easily learned. The mechanization of WLT through the rudder pedals was thought to be natural and was readily accepted by all evaluation pilots. The simulator motion provided realistic onsets of the lateral accelerations being commanded, but constraints on the simulator motion restricted the instantaneous lateral accelerations to $\pm 2.4 \mathrm{~m} / \mathrm{s}^{2}\left( \pm 8.0 \mathrm{ft} / \mathrm{s}^{2}\right)$.

\section{Wings-Level Turn}

Frequency

The matrix shown in Table 1 can be broken down into an evaluation of three underdamped $(\zeta<1)$, two overdamped $(\zeta>1)$, and one critically damped $(\zeta=1)$ configurations having the following transfer functions:

$$
\begin{array}{rlrl}
\frac{A_{y}}{\delta_{\mathrm{PED}}} & =\frac{K_{y} / 3.25}{s^{2} / \omega_{n}^{2}+2 \zeta s / \omega_{n}+1} & & \zeta<l \\
& =\frac{K_{y} / 3.25}{\left(s / \omega_{n}+I\right)^{2}} & \zeta=l \\
& =\frac{K_{y} / 3.25}{(s / a+l)(s / b+l)} & \zeta>l
\end{array}
$$

where $a$ and $b$ are the real roots of the quadratic equation (see Table 4).

Figure 4 shows the variation of pilot rating as a function of natural frequency, $\omega_{n}$, for the three underdamped cases $(\zeta=0.3,0.5,0.7)$, and Fig. 5 shows the same variation as a function of the low-frequency root, $a$, for the two overdamped cases $(\zeta=1.4,2.0)$. Since the ratings for the coarse task did not differ greatly from those for the fine task, only the fine-task data are shown in graphic form. The critically damped case is included in each figure for comparison. In all cases, as might be expected, the pilot ratings improved with increasing frequency for a given damping ratio, indicating that increased quickness of the response was favorable. However, it can be seen in Fig. 4 that there is considerable variation in pilot rating owing to the damping ratio, $\zeta$, for the underdamped cases, with the ratings improving with increased damping. Since the two overdamped cases are essentially first order,

$$
\frac{A_{y}}{\delta_{\mathrm{PED}}}=\frac{K_{y} / 3.25}{(s / a+I)(s / b+I)}
$$

and the high-frequency root, $b$, can generally be ignored; the pilot ratings are not affected by the damping ratio. Figure $S$ shows this variation clearly. Pilot ratings for the critically damped case are considerably worse than those for the overdamped cases and somewhat worse than the best un-

Table 4 Real roots of quadratic denominator $\left[(s+a)(s+b)=s^{2}+2 \zeta \omega_{n} s+\omega_{n}^{2}\right]$

\begin{tabular}{lcccccc}
\hline \hline$\omega_{n}$ & $\zeta$ & $a$ & $b$ & $\zeta$ & $a$ & $b$ \\
\hline 1 & 1.4 & 0.42 & 2.38 & 2.0 & 0.27 & 3.73 \\
2 & & 0.84 & 4.76 & & 0.54 & 7.46 \\
3 & & 1.26 & 7.14 & & 0.80 & 11.20 \\
4.5 & & 1.89 & 10.71 & & 1.21 & 16.79 \\
6 & 2.52 & 14.28 & & 1.61 & 22.39 \\
8 & & 3.36 & 19.04 & & 2.14 & 29.86 \\
10 & & 4.20 & 23.80 & & 2.68 & 37.32 \\
12 & & 5.04 & 28.56 & & 3.22 & 44.79 \\
15 & 6.30 & 35.70 & & 4.02 & 55.98 \\
19 & & 4.98 & 45.22 & & 5.09 & 70.91 \\
23 & & & & 6.16 & 85.84 \\
28 & & & & 7.50 & 104.50 \\
\hline \hline
\end{tabular}


derdamped case $(\zeta=0.7)$ for frequencies less than about 8 $\mathrm{rad} / \mathrm{s}$.

In general, pilot comments regarding these data indicate that the ratings are primarily related to the amount of lag in the system. The more apparent the lag, the worse the pilot rating. As the lag increases, the system response slows and it becomes more difficult to control the inputs without getting overshoots. In extreme cases, the pilot either cannot get the pipper over to the target or cannot stop it, once it is moving, without incurring large overshoots. The pilots generally seem to prefer quickness to damping and feel that they can overcome some lack of damping if the response is quick enough. However, there appears to be a limit to the amount of quickness and damping desired. For extreme cases of high damping and frequency, the pilots complained that the response was jerky and somewhat less than optimum. The very fast starting and stopping of the motion was disorienting.

\section{Bandwidth}

It was hypothesized that the pilot-rating data might better correlate on the basis of system bandwidth. For this purpose, bandwidth was first defined as the frequency at which there was a 3-dB drop in amplitude from the steady-state condition (see Table 1). Smooth variations of the average pilot ratings with this variable are seen (Fig. 6) for each damping ratio, with a distinctive progressive degradation in flying qualities accompanying a decrease in damping.

For the same test data, bandwidths were also calculated using the definition found in Ref. 6. Reference 6 defines bandwidth as the lowest frequency for which the open-loop phase margin is at least $45 \mathrm{deg}$ and the gain margin is at least 6 $\mathrm{dB}$. Figure 7 shows the correlation of average pilot rating with this alternative definition of bandwidth. There appears to be much better correlation in this case, with the data collapsing into a narrow band having a maximum scatter of $1 \frac{1 / 4}{4}$ rating

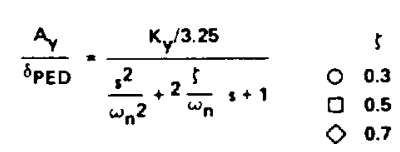

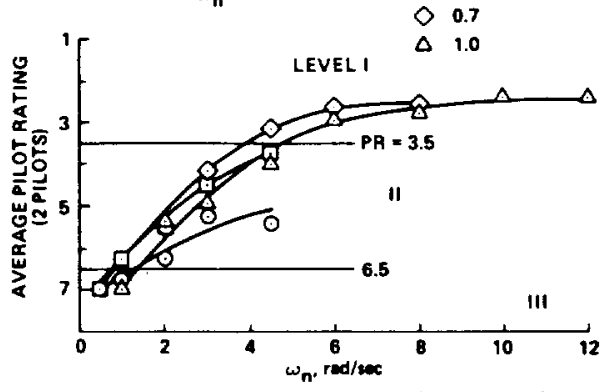

Fig. 4 Effect of natural frequency on pilot rating: fine task.

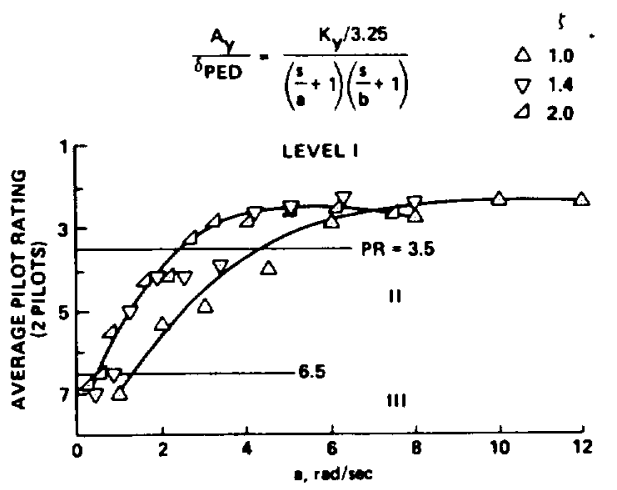

Fig. 5 Effect of low-frequency root on pilot rating: fine task. point. Figure 7 indicates minimum satisfactory $(P R=3.5)$ and minimum acceptable $(P R=6.5)$ bandwidths of approximately 2.3 and $0.5 \mathrm{rad} / \mathrm{s}$, respectively.

\section{Time Constant}

An evaluation of the pilots' recorded comments during the test program indicated that they were rating the airplane performance in the time domain rather than in the frequency domain, as their frequent references to lags and time delays attest. As a result, time constants were calculated for each test condition listed in Table 1. The time constant, in each case (underdamped, overdamped, and critically damped) was taken to be the time at which the response to a unit step input reached $63.2 \%$ of its steady-state value. For the underdamped oscillatory response the time constant was based on the envelope as calculated by the expression

$$
A_{y}=K_{y}\left(I-e^{-\zeta \omega_{n}{ }^{\prime}}\right)
$$

For the critically damped and overdamped cases the responses were given by

and

$$
A_{y}=K_{y}\left(l-e^{-\omega_{n} t}-\omega_{n} l e^{-\omega_{n}}\right)
$$

$$
A_{y}=K_{y}\left(1+\frac{a e^{-b t}-b e^{-a t}}{b-a}\right)
$$

respectively, where $a$ and $b$ are the real roots of the quadratic equation (Table 4). Pilot ratings are presented in Fig. 8 as a function of these time constants; they show excellent correlation for the fine-task data. (The pilot ratings for both tasks were nearly the same, differing by only about one-half a rating.) The time constants used and the average ratings for the two pilots are shown in Table 5 .

These data show that there is a minimum time constant $(0.15-0.2 \mathrm{~s})$ at which optimum performance of WLT is achieved and that level I (PR $\leq 3.5$ ) ratings were obtained for time constants less than about $0.4 \mathrm{~s}$ for the fine task and less than about $0.35 \mathrm{~s}$ for the coarse task. The WLT ratings became unacceptable at time constants greater than about 1.5

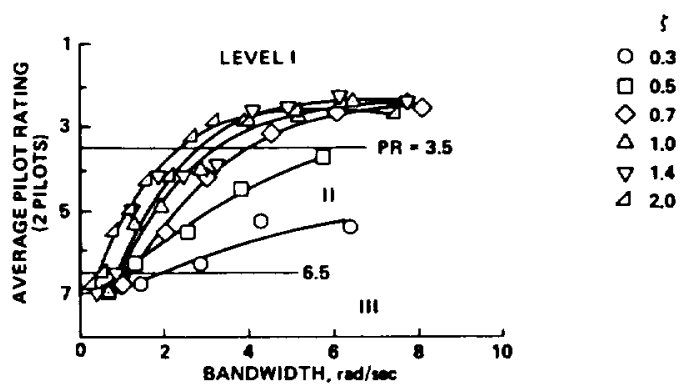

Fig. 6 Effect of bandwidth on pilot rating: fine task.

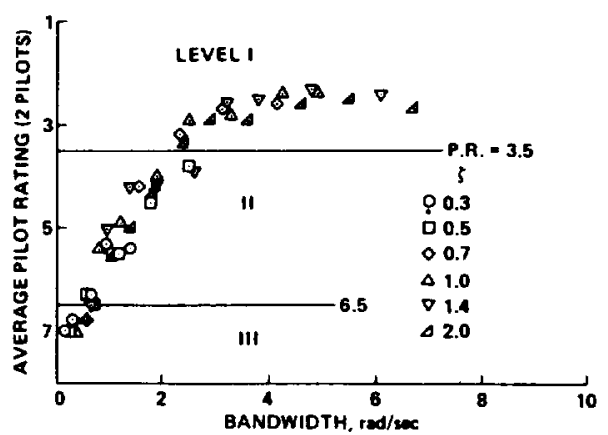

Fig. 7 Effect of bandwidth ${ }^{6}$ on pilot rating: fine task. 
Table 5 Equivalent time constants and average pilot ratings

\begin{tabular}{|c|c|c|c|c|c|c|c|c|c|c|c|c|c|c|c|c|c|c|}
\hline \multirow[b]{3}{*}{$\omega_{\prime \prime}$} & \multicolumn{18}{|c|}{$\zeta$} \\
\hline & \multicolumn{3}{|c|}{0.3} & \multicolumn{3}{|c|}{0.5} & \multicolumn{3}{|c|}{0.7} & \multicolumn{3}{|c|}{1.0} & \multicolumn{3}{|c|}{1.4} & \multicolumn{3}{|c|}{2.0} \\
\hline & $\tau$ & $C$ & $F$ & 7 & $C$ & $F$ & $\tau$ & $C$ & $F$ & $\tau$ & $C$ & $F$ & $T$ & $C$ & $F$ & $T$ & $C$ & $F$ \\
\hline 0.5 & 6.67 & 7 & 7 & 4.00 & 7 & 7 & & & & & & & & & & & & \\
\hline 1 & 3.33 & 7 & 6.8 & 2.00 & 6.5 & 6.3 & 1.43 & 7 & 6.8 & 2.15 & 7 & 7 & 2.84 & 7 & 7 & 3.98 & 7 & 6.8 \\
\hline 2 & 1.67 & 6.8 & 6.3 & 1.00 & 6 & 5.5 & 0.71 & 6.2 & 5.5 & 1.07 & 6.4 & 5.4 & 1.42 & 6.8 & 6.5 & 1.99 & 6.8 & 6.5 \\
\hline 3 & 1.11 & 5.8 & 5.3 & 0.67 & 5.7 & 4.5 & 0.48 & 4.8 & 4.2 & 0.72 & 5 & 4.9 & 0.95 & 5.3 & 5 & 1.33 & 6.2 & 5.5 \\
\hline 4.5 & 0.74 & 5.8 & 5.4 & 0.44 & 4 & 3.8 & 0.32 & 4.3 & 3.2 & 0.48 & 4.7 & 4 & 0.63 & 4.9 & 4.2 & 0.88 & 5.5 & 5 \\
\hline 6 & & & & & & & 0.24 & 3 & 2.7 & 0.36 & 3.8 & 2.9 & 0.47 & 4.3 & 4.2 & 0.66 & 5 & 4.3 \\
\hline 8 & & & & & & & 0.18 & 2.8 & 2.6 & 0.27 & 3 & 2.8 & 0.36 & 4 & 3.9 & 0.50 & 4.7 & 4.2 \\
\hline 10 & & & & & & & & & & 0.21 & 2.5 & 2.4 & 0.28 & 2.9 & 2.6 & 0.40 & 3.4 & 3.3 \\
\hline 12 & & & & & & & & & & 0.18 & 2.8 & 2.4 & 0.24 & 2.9 & 2.5 & 0.33 & 3 & 2.9 \\
\hline 15 & & & & & & & & & & & & & 0.19 & 2.4 & 2.3 & 0.27 & 3.2 & 2.9 \\
\hline 19 & & & & & & & & & & & & & 0.15 & 2.8 & 2.4 & 0.21 & 2.8 & 2.6 \\
\hline 23 & & & & & & & & & & & & & & & & 0.17 & 2.8 & 2.5 \\
\hline 28 & & & & & & & & & & & & & & & & 0.14 & 2.9 & 2.7 \\
\hline
\end{tabular}

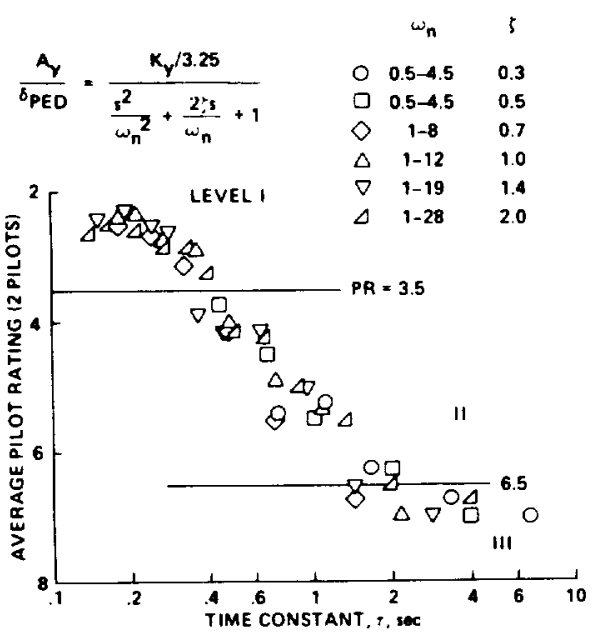

Fig. 8 Effect of response time constant on pilot rating: fine lask.

s. These results agree with the pilot comments that the lag of the system was the most important factor in determining the pilot ratings. As previously mentioned, the pilots felt that they could tolerate some lack of damping if the response was quick enough, but that if the response was too quick the performance became jerky and disorienting and the flying qualities deteriorated. The slight break in the curves (Fig. 8) at the low time constants is indicative of this degradation.

The data of Figs. 7 and 8 suggest that a criterion for WLT maneuvering could justifiably be based on either response time constant or bandwidth (as defined in Ref. 6). The minimum satisfactory $(P R=3.5)$ values of time constant and bandwidth appear to be consistent (i.e., reciprocals of each other). The same statement can be made for the minimum acceptable $(P R=6.5)$ values.

\section{Transport Delay}

Tests were conducted to examine the effect of adding transport delay to a system otherwise having good characteristics (see Table 2), assuming the transfer function to be of the form

$$
\frac{A_{y}}{\delta_{\mathrm{PED}}}=\frac{\left(K_{y} / 3.25\right) e^{-A s}}{(s / a+1)(s / b+1)}
$$

where $\zeta=1.4, a=6.30, b=35.70$, and $\omega_{n}=15$.

Figure 9 shows the average pilot ratings of the two pilots as functions of transport delay. These data show that the ad-

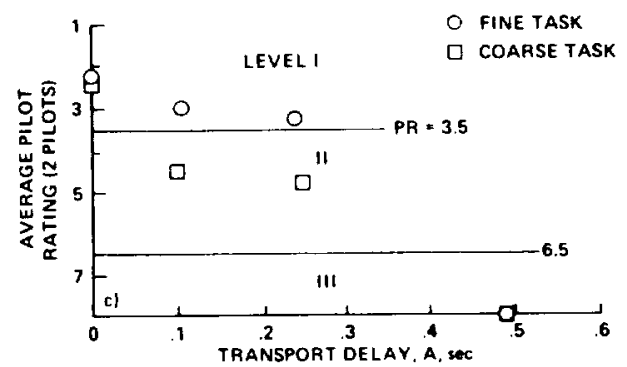

Fig. 9 Effect of Iransport delay on pilot rating.

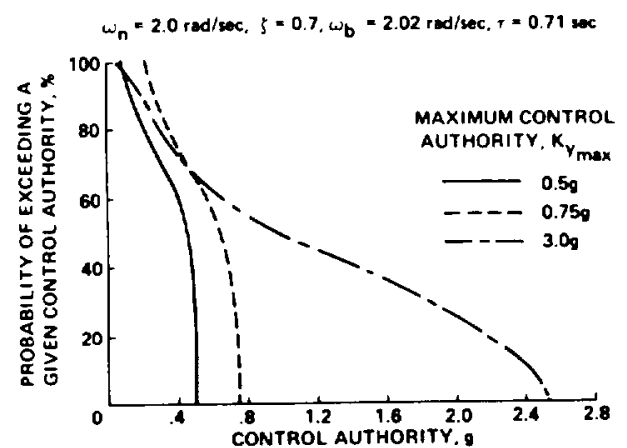

Fig. 10 Cumulative frequency distribution of commanded side acceleration: pllot $\mathrm{C}$.

dition of even small amounts of delay degrades the system response. This agrees with previous findings that the more lag in the system the more difficult the tracking task becomes.

\section{Conirol Authority}

Early in the investigation, pilots were provided with three levels of side-force control authority 10 perform the established dive-bombing task and to evaluate the amount of control required for WLT maneuvering. These levels corresponded to maximum commanded side accelerations of $0.5,0.75$, and $3.0 \mathrm{~g}$. The $3.0 \mathrm{~g}$ level was included to insure sufficient control for the task and was treated as being essentially unlimited. The control system was mechanized to give full pedal travel for each one of these authorities.

The results of this control authority study are shown in Fig. 10 for a configuration having a damping ratio of 0.7 and a frequency of $2 \mathrm{rad} / \mathrm{s}$ (see Table 3). The curves are cumulative distributions of the lateral accelerations used during the coarse-tracking maneuver, calculated over the time interval 


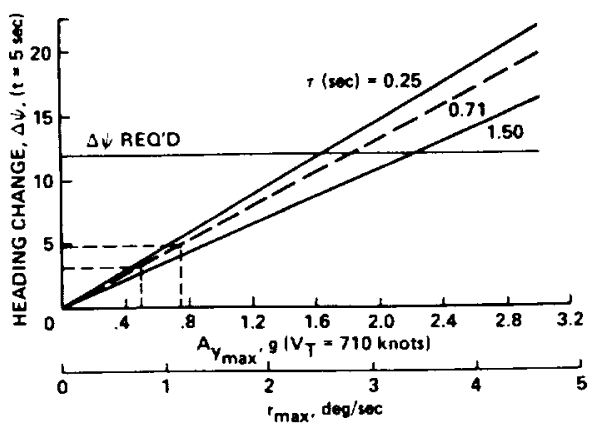

Fig. 11 Control power and time response required for a particular heading change, $\Delta \downarrow$.

beginning with the first significant change of side acceleration after the target-change signal and ending with bomb release. An analytical prediction of the control power and time response required to make a particular heading change based on the second-order model used in this investigation is shown graphically in Fig. 11. A maneuvering time of $5 \mathrm{~s}$ and a release velocity of 710 knots were assumed; curves are presented for response-time constants of $0.25,0.71$ (same as for Fig. 10), and $1.50 \mathrm{~s}$. This figure and Fig. 10 are considered together in the following discussion. Because of the demands placed upon the WLT maneuvering system by the magnitude of the maneuver (12-deg heading change in $5 \mathrm{~s}$ ), the two lowest authorities $(0.5$ and $0.75 g)$ proved insufficient to perform the coarse task in the time available. With $0.5 \mathrm{~g}$ maximum, even the fine task could not be accomplished unless the pilot happened to roll out nearly on target. Figures 10 and 11 show the inadequacy of the 0.5 and $0.75 \mathrm{~g}$ authority levels by the high probability of exceeding, say, $90 \%$ of the authority available and by the low predicted values of heading change, $\Delta \psi$, compared with that required for the maneuver. Both figures, however, show the $3 g$ level to be more than adequate for the task. At this level, time histories showed typically that a maximum of $2.5 \mathrm{~g}$ was commanded (and then only momentarily), and that the maneuver was completed in time to do some fine tracking. If one were to select a minimum authority to perform the maneuver, Figs. 10 and 11 indicate that a value of approximately $1.6-2.2 \mathrm{~g}$ would be adequate, depending on the time constant.

Although the magnitude of the coarse-tracking maneuver investigated in this simulation may be substantially greater than necessary for normal air-to-ground operations, and impractical from the standpoint of current aircraft design practice, the general agreement between the experimental results (Fig. 10) and analytical predictions (Fig. 11) suggests that such predictions of control authority required for WLT maneuvers may be made to suit the parameters of any air-toground task similar to the present one, and can aid in arriving at a first estimate of side acceleration authority required for a given response time constant.

\section{Comparison with Conventional Airplane}

Each of the Eglin AFB pilots flew both the fine and coarse tasks with the conventional aircraft. Individual pilot ratings for the coarse task were in close agreement, and the task was rated as being either nearly impossible $(P R=6)$ or impossible $(P R=7)$. Although the basic aircraft had good flying qualities (similar to those of an F-15), the control authority of the aircraft and the pendulum effect of the pipper made it nearly impossible to bank the airplane, make the necessary lateral correction, and level out on the target in the time available. It should be remembered that the task was made particularly difficult in order that the advantages or disadvantages of the different control modes would become obvious. This performance of the basic aircraft illuminates the benefits of
WLT, for we have shown previously that the task was easily accomplishable $(P R=2)$ when using WLT with good response characteristics.

The agreement among pilot ratings by each pilot for the fine task was not as good as that for the coarse task. One pilot felt that the aircraft damping and control sensitivity were good and that he could accomplish the task with a minimum of compensation. He gave this case a pilot rating of 3 . The second pilot felt the fine task to be difficult, but that it could be done easily with a lot of guesswork as to the amount of bank to put in and take out (pendulum effect); he gave the fine task a rating of 5 .

It was the general feeling of both pilots that WLT with good response characteristics was a significant improvement over the basic aircraft. WLT greatly simplified the lateral tracking task and allowed more attention to be devoted to the longitudinal task than was possible in the basic aircraft.

\section{Concluding Remarks}

Piloted six-degrees-of-freedom motion simulator investigations conducted at Ames Research Center demonstrated that the WLT control mode was very useful in decreasing pilot workload during an air-to-ground weapon delivery task and in improving airplane flying qualities in comparison with those of a conventional aircraft, particularly if any significant amount of heading change was necessary to acquire the target.

The parametric evaluation of the frequency and damping requirements for the WLT control mode has shown that pilot ratings for various combinations of damping ratio and frequency response correlate extremely well on the basis of the time required for the lateral-acceleration response to a unit step input to reach $63.2 \%$ of its steady-state value. This time constant correlated the data for either underdamped, overdamped, or critically damped responses.

The data show that pilot ratings improved with decreased time constant (response is quickened), but that there is a minimum time constant $(\approx 0.15 \mathrm{~s})$ for optimum performance. Decreasing the time constant below the minimum results in a system that is too quick, resulting in degraded ratings because of jerkiness and disorientation. In general, time constants less than about $0.4 \mathrm{~s}$ resulted in pilot ratings of 3.5 or better for both the fine and coarse tasks.

The data also show extremely good correlation on the basis of bandwidth, defined as the lowest frequency for which the open-loop phase margin is at least $45 \mathrm{deg}$ and the gain margin is at least $6 \mathrm{~dB}$. The critical values (minimum satisfactory or minimum acceptable) of time constant and bandwidth, as defined in this paragraph, appear to be consistent.

The addition of a transport delay to a basically good system degraded both the performance and the pilot ratings. Most pilot comments regarding degradation in performance pertained to various amounts of transport delay or lag in the system. Only for cases having low damping and lowfrequency response did oscillatory motion become a problem. For cases having high-frequency response, even with high damping, the problem became one of excessive quickness.

The side-acceleration control authority required to perform a coarse change of target task was also assessed. For a largedisturbance maneuver (12-deg heading change in $5 \mathrm{~s}$ ), sideacceleration capability of the order of $1.6-2.2 \mathrm{~g}$ was required, based on cumulative frequency distributions of control usage and analytical predictions of WLT maneuvering capability. Although the coarse task selected for the present study is quite severe in view of normal tactical requirements and current aircraft design practice, agreement between the experimental results and analytical predictions suggests that the prediction method can aid in arriving at a first estimate of required sideacceleration authority. 


\section{References}

'Carlson, E.F., "Direct Sideforce Control for Improved Weapon Delivery Accuracy," AlAA Paper 74-70, 1974.

${ }^{2}$ Swortzel, F.R. and Barfield, F.A., "The CCV Fighter Program-Demonstrating New Control Methods for Tactical Aircraft," AIAA Paper 76-889, 1976.

3 Brulle, R.V., Moran, W.A., and Marsh, R.G., "Direct Side Force Control Criteria for Dive Bombing." AFFDL-TR-76-78, Vols. 1 and II, Sept. 1976.
${ }^{4}$ Sinacori, J.B., Stapleford, R.L., Jewell, W.F., and Lehman, J.M., "Researcher's Guide to the NASA-Ames Flight Simulator for Advanced Aircraft (FSAA)," NASA CR-2875, 1977.

"Cooper, G.E. and Harper, R.P. Jr., "The Use of Pilot Rating in the Evaluation of Aircraft Handling Qualities," NASA TN D-5153, 1969.

${ }^{6}$ Hoh, R.H., Myers, T.T., Ashkenas, I.L., Ringland, R.F., and Craig, S.J., "Development of Handling Quality Criteria for Aircraft with Independent Control of Six Degrees of Freedom," AFWAL-TR. 81-3027, April 1981. 